\title{
TRATAMIENTO INMUNOSUPRESOR SISTÉMICO Y TRASPLANTE DE MEMBRANA AMNIÓTICA EN ESCLERITIS NECROTIZANTE INDUCIDA POR CIRUGÍA (ENIC)
}

\author{
SUCCESFUL TREATMENT OF SURGICALLY INDUCED \\ NECROTIZING SCLEROKERATITIS (SINS) WITH SYSTEMIC \\ IMMUNOSUPPRESIVE AGENTS AND AMNIOTIC MEMBRANE \\ GRAFTING
}

\author{
CORDERO-COMA M ${ }^{1}$, FRANCO-BENITO M ${ }^{1}$, GARCÍA-RUIZ-DE-MORALES JM ${ }^{2}$, \\ ALONSO-ORCAJO N ${ }^{3}$, DEL BARRIO-MANSO I ${ }^{4}$
}

\begin{abstract}
RESUMEN
Caso clínico: Presentamos el caso de una mujer de 74 años que desarrolló una escleroqueratitis necrotizante en su ojo izquierdo tras cirugía de catarata no complicada. No tenía antecedentes de enfermedad autoinmune sistémica. El análisis histopatológico de la lesión mostró granulomatosis necrotizante con abundantes células plasmáticas.

Discusión: La escleroqueratitis necrotizante inducida por cirugía (ENIC) es una grave afección que requiere tratamiento precoz y agresivo tratando de prevenir sus potencialmente devastadoras consecuencias. La resección conjuntival junto con implante de membrana amniótica pueden ser necesarias para interrumpir temporalmente el proceso inmunológico local. No obstante, es esencial asociar un tratamiento inmunosupresor sistémico.
\end{abstract}

\begin{abstract}
Clinical case: We report the case of a 74-year-old female who developed a necrotizing sclerokeratitis affecting her left eye after uncomplicated cataract surgery. She had no previous history of systemic autoimmune disease. Histopathology of the lesion revealed necrotic granulomatosis with an increased number of plasma cells.

Discussion: Surgically induced necrotizing sclerokeratitis (SINS) is a serious entity which requires prompt and aggressive therapy to prevent its potential devastating ocular consequences. Conjunctival resection and amniotic membrane grafting may be necessary to temporarily interrupt local immunologic events in severe cases. However, associated systemic immunomodulatory therapy seems to be mandatory (Arch Soc Esp Oftalmol 2009; 84: 577-580).
\end{abstract}

\footnotetext{
Recibido: 10/1/08. Aceptado: 4/11/09.

Hospital de León. León. España.

1 Licenciado en Medicina. Servicio de Oftalmología. Unidad de Uveítis.

2 Doctor en Medicina. Sección de Inmunología. Unidad de Uveítis.

3 Licenciado en Medicina. Servicio de Anatomía Patológica.

4 Licenciado en Medicina. Servicio de Oftalmología.

Comunicación presentada en el LXXXI Congreso de la S.E.O. (Zaragoza 2005).

Correspondencia:

Miguel Cordero-Coma

Hospital de León

C/. Altos de Nava, s/n

24080 León

España

E-mail: miguelcorderocoma@gmail.com
} 
Palabras clave: Escleritis necrotizante inducida por cirugía (ENIC), trasplante de membrana amniótica, agentes inmunosupresores, inmunomodulación, complicaciones tras cirugía de catarata, uveítis.
Key words: Surgically induced necrotizing scleritis, amniotic membrane grafting, immunosuppressive agents, immunomodulation, complications after cataract surgery, uveitis.

\section{INTRODUCCIÓN}

La escleritis necrotizante inducida por cirugía (ENIC) es una rara afección autoinmune local que ocurre próxima a incisiones quirúrgicas previas y ha sido relacionada con prácticamente todo tipo de cirugías oculares (1-5). La ENIC se desarrolla tras un período de latencia que podría oscilar entre algunas semanas y varios años, y puede ser la primera manifestación de una vasculitis autoinmune o puede indicar el inicio de una vasculitis en un paciente ya diagnosticado de alguna entidad autoinmune (2). Las infecciones post-quirúrgicas así como el uso de determinados materiales de sutura han sido identificados como factores potencialmente contribuyentes al desarrollo de ENIC (3).

\section{CASO CLÍNICO}

Una mujer sana de 74 años acude referida por su oftalmólogo para evaluación y tratamiento de ojo rojo doloroso de aproximadamente un mes de evo-

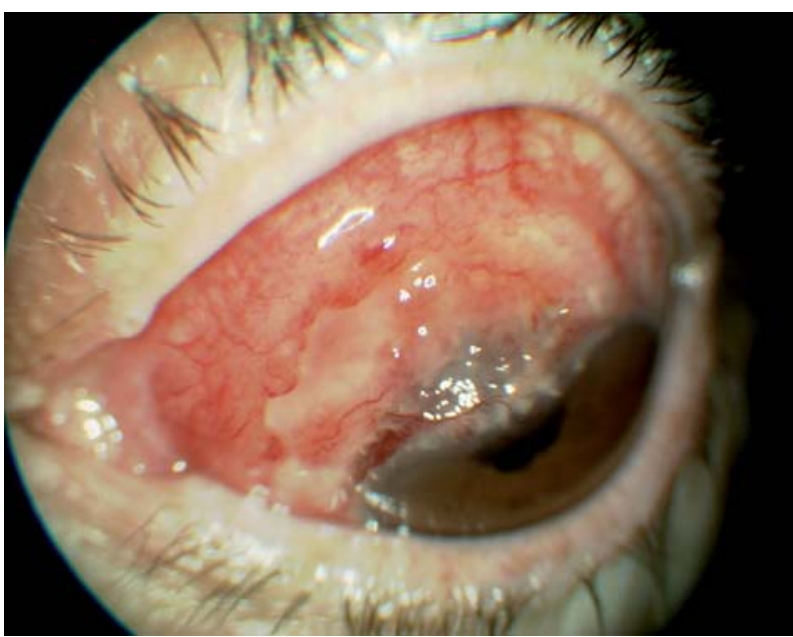

Fig. 1: Visión a la lámpara de hendidura del ojo izquierdo en su primera visita. Obsérvese la lesión sobreelevada a nivel del limbo superonasal con ingurgitación vascular superficial y profunda, así como microinfiltrados corneales. lución. No tenía antecedentes de interés, a excepción de cirugía de catarata no complicada (facoemulsificación) en ese mismo ojo unos cuatro meses antes. La exploración oftalmológica reveló una lesión sobreelevada a nivel del limbo superonasal de su ojo izquierdo (próxima a la incisión quirúrgica) asociada a múltiples microinfiltrados corneales, así como ingurgitación vascular conjuntival y del plexo escleral profundo (fig. 1). Se inició tratamiento con colirios antibióticos de amplio espectro, añadiéndose además instilaciones de colirios de suero autólogo y medroxiprogesterona.

Debido a la escasa respuesta al tratamiento, se solicitaron diversas pruebas serológicas (incluyendo bioquímica, hemograma, reactantes de fase aguda y marcadores de autoinmunidad). Paralelamente se inició tratamiento con corticoides sistémicos a dosis de $1 \mathrm{mg} / \mathrm{kg}$. Además decidimos tomar una muestra escleroconjuntival, previa resección de la conjuntiva adyacente, con posterior implante de membrana amniótica (técnica «inlay») unido a la aplicación de adhesivo biológico (Fibrin Glue ${ }^{\circledR}$ ) sobre el área de lesión (fig. 2). La biopsia (figs. 3a y 3b) fue diagnós-

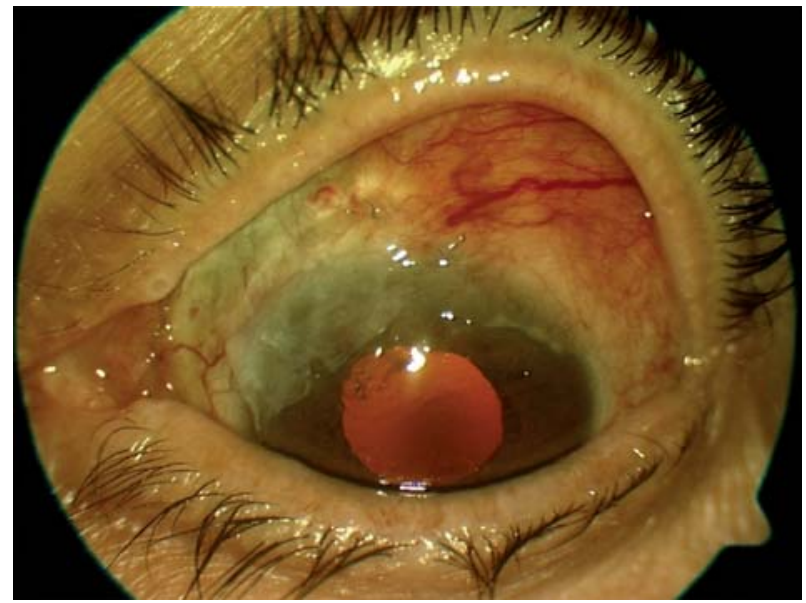

Fig. 2: Aspecto del mismo ojo tras la resección de conjuntiva adyacente e implante de membrana amniótica. Obsérvese la mejoría en el área previamente inflamada tras la cirugía y el empeoramiento del tejido anexo superotemporal donde la reacción inflamatoria continúa. 
tica de granulomatosis necrotizante asociada a hiperplasia de células plasmáticas sin indicios de vasculitis acompañante. Los resultados de las pruebas serológicas realizadas fueron normales.

Considerando la posibilidad de ENIC, se decidió instaurar tratamiento con Ciclosporina A (3 $\mathrm{mg} / \mathrm{kg} / \mathrm{día}$ ) unido a los corticosteroides orales $(1 \mathrm{mg} / \mathrm{kg} / \mathrm{día})$, pero unas ocho semanas tras su inicio el cuadro inflamatorio persistía y, dada su severidad, se decidió sustituir Ciclosporina por Azatioprina (100 mg/día) manteniendo los corticosteroides orales.

Este régimen terapéutico consiguió progresivamente controlar el proceso inflamatorio (figs. 4a y 4b). Siete meses después del inicio del cuadro la paciente permanece asintomática y continúa con el tratamiento.

\section{DISCUSIÓN}

El diagnóstico de ENIC en el caso que presentamos se basó fundamentalmente en los siguientes hallazgos:
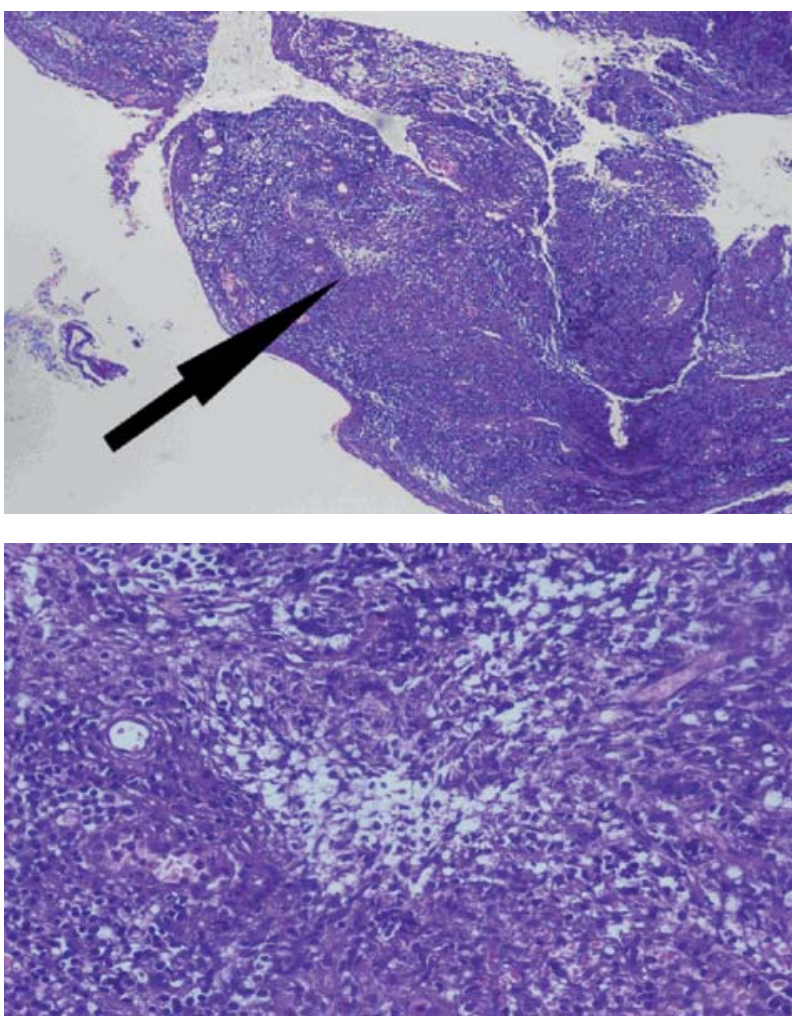

Fig. 3: (A) Biopsia del tejido resecado (Tinción con hematoxilina/eosina, amplificación $2 X$ ) mostrando focos de granulomatosis necrotizante, que se aprecian mejor en la imagen $(B)$ con amplificación $10 X$.
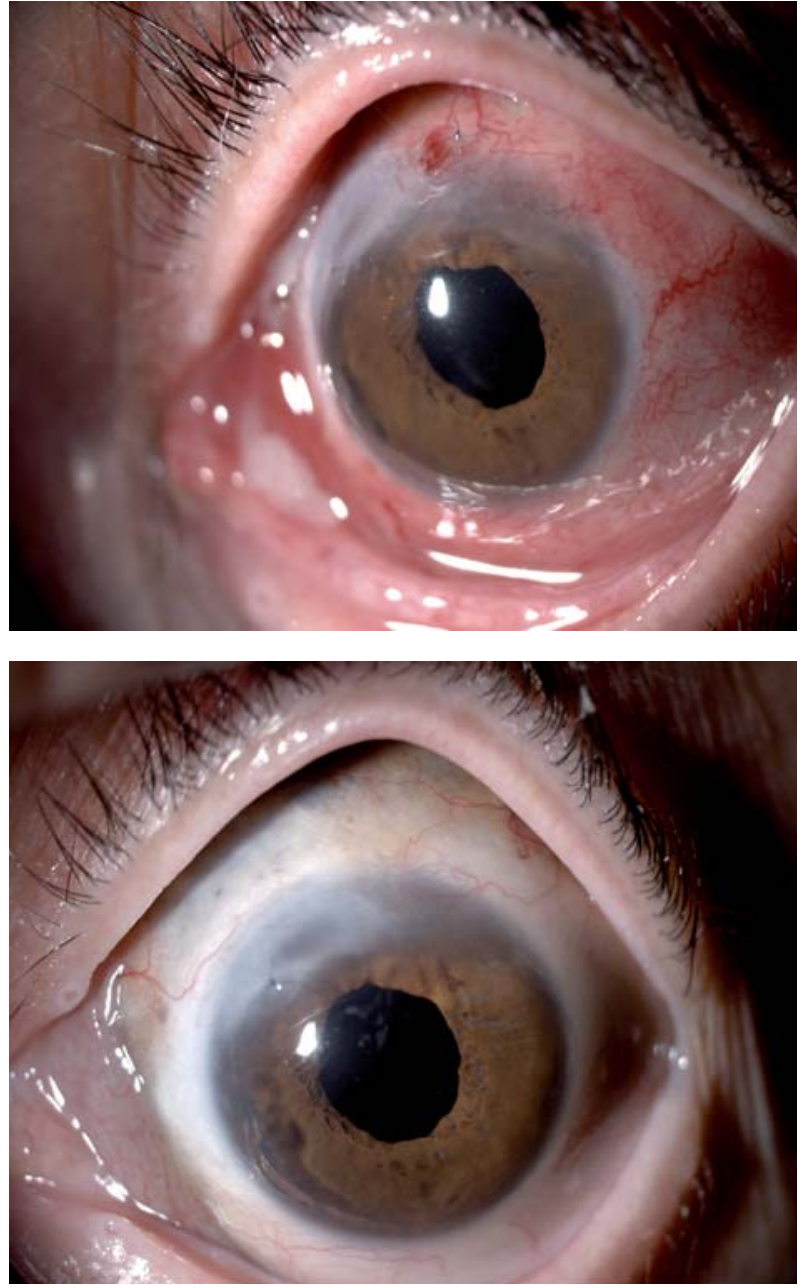

Fig. 4: Visión a la lámpara de hendidura 1 mes y 3 meses (figuras $A$ y $B$ respectivamente) tras la introducción de Azatioprina. Obsérvese la ausencia de ingurgitación vascular e infiltrados corneales, así como el adelgazamiento residual de la esclera afecta.

1) Antecedentes de cirugía ocular.

2) Ausencia de signos clínicos o serológicos de enfermedad autoimmune sistémica.

3) Desarrollo de una escleritis necrotizante próxima a la herida quirúrgica previa.

Creemos que el tratamiento quirúrgico, aunque paliativo y transitorio, es un importante adyuvante en el tratamiento de estos pacientes. La resección de la conjuntiva adyacente al área de lesión ha sido descrita como una técnica eficaz para la disminución de la concentración de proteasas (tales como la colagenasa), así como para la interrupción temporal del proceso inflamatorio local, disminuyendo el aporte de citoquinas pro-inflamatorias (4). En el 
caso que nos ocupa, la membrana amniótica contribuyó al control de la inflamación en el área del implante, si bien las áreas adyacentes comenzaron a afectarse, dado que la cirugía por sí sola no es capaz de detener el proceso inmunológico sistémico subyacente. Por todo ello se decidió iniciar un tratamiento inmunomodulador tratando de conseguir un óptimo control de la enfermedad y de prevenir futuras recurrencias.

Se ha postulado que la ENIC podría relacionarse con una isquemia relativa localizada asociada a la herida quirúrgica (1). En aproximadamente un $60 \%$ de los casos se identificó una patología responsable del cuadro, siendo en su mayoría una enfermedad del tejido conectivo. Sin embargo, poco se conoce acerca del mecanismo inmunológico que induce su aparición. Se ha especulado que podría tratarse de una reacción de hipersensiblidad retardada Tdependiente dirigida frente a ciertos antígenos tisulares no identificados (fruto del trauma quirúrgico y/o de la isquemia temporal) (1). En este sentido algunos autores (5) han obtenido resultados prometedores usando agentes específicamente inhibidores de la respuesta T-celular como el Tacrolimus. Paradójicamente, en nuestro caso, la paciente respondió satisfactoriamente a Azatioprina (que inhíbe las respuestas inmunológicas $\mathrm{T}$ y $\mathrm{B}$ dependientes) y no a Ciclosporina A (un T-inhibidor). De hecho en nuestro paciente la biopsia mostró hiperplasia de células plasmáticas en torno al área lesional, sugiriendo una implicación preferente de la respuesta celular B-dependiente. En este sentido, aunque no se han identificado autoanticuerpos, sí se ha demostrado la presencia de inmunocomplejos en algunos casos (1), reforzando la hipótesis de una implicación de la respuesta B-celular. El hecho de que la ENIC sea o no una entidad clínica definida o sólo la primera manifestación o el «trigger» de un proceso autoinmune en ciertos individuos susceptibles es aún objeto de controversia.

\section{BIBLIOGRAFÍA}

1. O'Donoghue S, Lightman S, Tuft S, Watson P. Surgically induced necrotising sclerokeratitis (SINS)_precipitating factors and response to treatment. Br J Ophthalmol 1992; 76: $17-21$.

2. Sainz de la Maza M. Scleritis. In: Foster CS, Azar DT, Dohlman CH. Smolin and Thoft's: The Cornea, Scientific Foundations and Clinical Practice (4th Edition). Philadelphia: Lippincott Williams \& Wilkins, 2005; 559-569.

3. Beatty S, Chawdhary S. Necrotizing sclerokeratitis following uncomplicated cataract surgery. Acta Ophthalmol Scand 1998; 76: 382-383.

4. Lambiase A, Sacchetti M, Sgrulletta R, Coassin M, Bonini $S$. Amniotic membrane transplantation associated with conjunctival peritomy in the management of Mooren's ulcer: a case report. Eur J Ophthalmol 2005; 15: 274-276.

5. Young AL, Wong SM, Leung AT, Leung GY, Cheng LL, Lam DS. Successful treatment of surgically induced necrotizing scleritis with tacrolimus. Clin Exp Ophthalmol 2005; 33: 98-99. 